\title{
BRAF Mutation Correlates with Aggressive Features, Little Predictive Value
}

\author{
${ }^{1}$ Andrew Quong, ${ }^{2}$ Colin Wynne, ${ }^{3}$ Joseph Curry, ${ }^{4}$ Kathryn Scott, ${ }^{5}$ David Rosen, ${ }^{6}$ David Cognetti \\ ${ }^{7}$ Edmund Pribitkin, ${ }^{8}$ Elizabeth Duddy
}

\begin{abstract}
Objective: With widespread and sophisticated imaging techniques, micro papillary thyroid cancers (PTCs) may be discovered prior to developing the classic pathological determinants of aggressiveness, such as extrathyroidal extent (ETE). Many studies have suggested that the V600E B-Raf proto-oncogene (BRAF) mutation can be used as a marker for aggressive disease. One objective of this study is to determine what prognostic value this mutation holds. However, nearly all of current studies have focused solely on classically aggressive tumors, not classically nonaggressive samples. This study also seeks to determine the BRAF mutation status in both the groups of tumors.
\end{abstract}

Materials and methods: Sixty-six PTC samples were tested for the V600E BRAF mutation using competitive allele-specific TaqMan probes in real-time PCR (Applied Biosystems/Life Technologies). Testing demonstrates that this assay has at least a $<5 \%$ sensitivity to the mutation. Forty-five samples had at least one of four aggressive features. Samples with vascular invasion, ETE or lymph node metastasis (LNM) were also characterized as having poor prognosis.

Results: The V600E BRAF mutation was found in 27 of the 45 aggressive samples $(60.0 \%)$ and 5 of the 21 nonaggressive samples. The Fisher exact test resulted in a correlation between aggressiveness and BRAF mutation as well as correlations between ETE, LNM and the BRAF mutation. When using the BRAF mutation as a predictor of prognosis based on the pathological features of aggressiveness, there was $60 \%$ sensitivity and $80 \%$ specificity.

Conclusion: The V600E BRAF mutation is correlated with pathological aggressive features, but may lack sufficient specificity or sensitivity to be used as a marker to predict outcome.

Keywords: Papillary thyroid cancer, BRAF, Molecular diagnostics, Proteomics.

How to cite this article: Quong A, Wynne C, Curry J, Scott $\mathrm{K}$, Rosen D, Cognetti D, Pribitkin E, Duddy E. BRAF Mutation Correlates with Aggressive Features, Little Predictive Value. Int J Head Neck Surg 2014;5(3):130-134.

\footnotetext{
${ }^{1}$ Associate Professor, ${ }^{2}$ Postdoctoral Fellow, ${ }^{3,5,6}$ Assistant Professor, ${ }^{4}$ Researcher, ${ }^{7}$ Professor, ${ }^{8} \mathrm{RN}$ and $\mathrm{BSN}$

1,2,4 Department of Cancer Biology, Thomas Jefferson University Philadelphia, USA

${ }^{3,5-8}$ Department of Otolaryngology, Thomas Jefferson University Philadelphia, USA
}

Corresponding Author: Andrew Quong, Associate Professor Department of Cancer Biology, Bluemle Life Sciences Building 2335, 10th Street, Room 311, Philadelphia, PA 19107, USA e-mail: andrew.quong@kimmelcancercenter.org

\section{Source of support: Nil}

Conflict of interest: None

\section{INTRODUCTION}

The increasing incidence ${ }^{1}$ and earlier diagnosis of papillary thyroid cancer (PTC) have prompted a search for prognostic indicators apart from the traditional histological correlates of aggressiveness. Advances in ultrasonographic screening and aspiration biopsy allow clinicians to detect concerning nodules earlier, including micro PTC. Given the very favorable prognosis of PTC overall, difficulties arise in distinguishing which micro PTC require aggressive treatment and which do not. ${ }^{2,3}$ To that end, much current research effort focuses on accurate and reliable markers for aggressive behavior in such lesions.

Traditionally, there are four pathological features for aggressive behavior in PTC. Capsular invasion (CI) is tumor growth through the lymph node capsule. ${ }^{4} \mathrm{~A}$ potentially more ominous second criterion is extrathyroidal extension (ETE) of the primary tumor into the surrounding tissues. Multiple foci of vascular invasion are also a feature of aggression. Regional lymph node metastasis (LNM) is a sign of aggressive behavior in patients over 45 years of age. Each of these features may require additional treatment, such as more extensive surgery (e.g. functional neck dissection) or radioactive iodine.

BRAF is a serine/threonine kinase and a part of the mitogen activated protein kinase/extracellular signalrelated kinase (MAPK/ERK) signaling pathway. This pathway plays a role regulation of cell cycle, differentiation and lysosomal processing. ${ }^{5}$ Commonly mutations of B-Raf proto-oncogene (BRAF) occur at exon 15, which leads to constitutive activation and subsequent oncogenesis. ${ }^{6}$ The most common mutation is a tyrosine to adenine switch at base 1799 , which changes that reading frame to produce a glutamic acid residue at position 600 rather than a valine residue (V600E). Research has shown this mutation plays a critical role in melanoma, ${ }^{7}$ colorecta $^{8}$ and thyroid cancers. In mice, the V600E mutation can lead to more tumor-associated macrophages as well as epithelial to mesenchymal transition, allowing increased migration, and thus a higher likelihood of invasion or metastasis. ${ }^{9}$ In the thyroid, researchers have shown that 
the presence of BRAF mutation in nodules is associated with malignancy, while BRAF negative biopsies were found to be benign. ${ }^{10-12}$ However, the utility of BRAF mutation status to stratify patients concerning more aggressive clinical behavior and thus more aggressive therapy is unclear. A meta-analysis by Xing in $2010^{13}$ assessed 19 studies reviewing a correlation between BRAF mutation and ETE and 26 studies reviewing a correlation between BRAF mutation and LNM. Of the ETE studies, only $7(36.8 \%)$ found a statistically significant correlation. Of the studies on BRAF mutation and LNM, only $7(26.9 \%)$ found a statistically significant correlation. While a number of contrary studies exist, ${ }^{14-17}$ a number of additional studies suggest a correlation between BRAF and these two features. Basolo et al examined 1060 tumors less than $2 \mathrm{~cm}$, found the BRAF mutation in $44 \%$ of those samples, and showed strong correlation between both features and BRAF mutation. ${ }^{18}$ Kim et al studied 547 PTC of all sizes and found the BRAF mutation in $64 \%$ of samples with ETE. ${ }^{19}$ Howell et al, in 2011, looked at 219 PTCs ranging from 0.2 to $5 \mathrm{~cm},{ }^{20}$ finding $39 \%$ of these samples contained the BRAF mutation, and $77 \%$ with ETE contained BRAF mutation.

An updated meta-analysis by Kim selected 27 studies including 5655 patients and concluded that PTC patients with the BRAF (V600E) mutation had increased odds ratios of extrathyroidal invasion, lymph node metastasis and an advanced TNM stage. ${ }^{21}$

According to National Cancer Institute Surveillance, Epidemiology and End Results (NCI-SEER) statistics, patients with PTC with LNM, ETE, or vascular invasion have a 10 years survival rate between 91 and $94 \%$ (Graph 1). While this may seem high, it is much lower than the $98.4 \%$ relative survival rate of all PTC patients or the 99.6\% 10 years survival rate of PTC patients without any

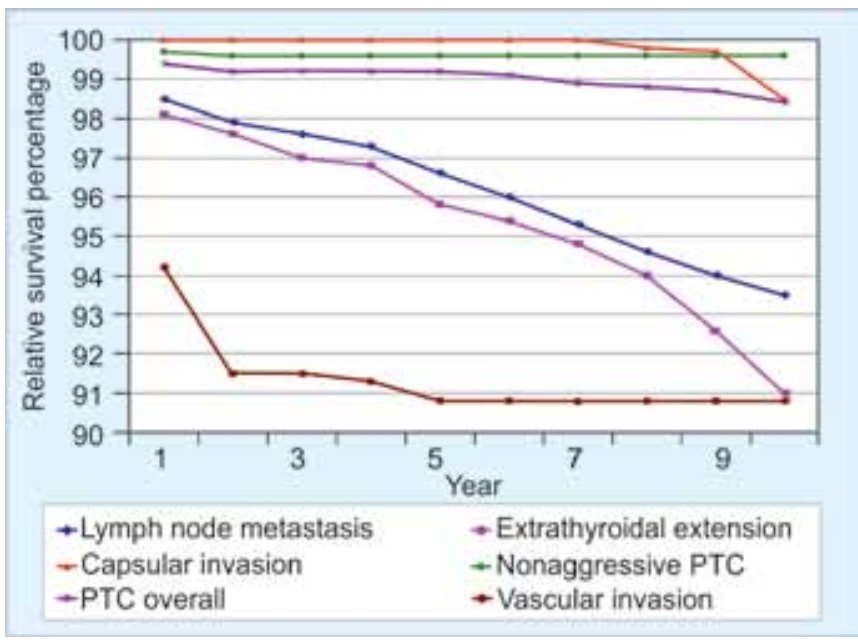

Graph 1: NCl-SEER survival data for pathological features of clinical aggressiveness. LMN, ETE, and vascular invasion all show poor prognosis with relative survival falling below $95 \%$ over 10 years of the aggressive features. In lieu of detailed follow-up data from patients in this study, these three pathological features can be used as a surrogate for poor prognosis.

The goal of this study was to further characterize the role of BRAF V660E in classically aggressive pathologic features compared to other nonaggressive tumors, and determine whether testing for this mutation should be used as a prognostic indicator in surgical and therapeutic decisions.

\section{MATERIALS AND METHODS}

This protocol was reviewed and approved by our institutional IRB. Sixty-six thyroid samples were collected and were cut into $20 \mu \mathrm{m}$ sections and the deoxyribonucleic acid (DNA) was extracted using the chemagic magnetic separation module extraction robot (Chemagen). DNA was quantified using the NanoDrop 2000. BRAF mutation status was analyzed by real-time PCR using castPCR BRAF assays (Life Technologies). V600E mutant and wild type BRAF mutation assays were used for each sample. Twenty ng of DNA was used for each assay. Internal positive controls were also added to each assay, using exogenous DNA and a different fluorescent dye. Statistical testing was done using the Fisher exact test to determine a correlation between V600E BRAF mutation and the aggressive features. Samples were divided into 'aggressive' or 'nonaggressive' groups. Forty-five of the 66 samples were labeled aggressive by meeting at least one of the four features of ETE, LNM, CI, or vascular invasion; the remaining 21 were labeled nonaggressive.

\section{RESULTS}

BRAF mutation positivity was found in 27 of the 45 aggressive samples $(60.0 \%)$. Five of 21 nonaggressive samples were positive for the BRAF mutation (23.8\%). Using the Fisher exact test, a significant correlation was demonstrated between these aggressive features and the BRAF mutation with a p-value less than 0.007. Individually, only LNM and ETE were significantly correlated with the mutation. Over $75 \%$ of the samples with extrathyroidal extension were found to carry the BRAF mutation $(p=0.0022)$, and over $65 \%$ of the samples with lymph node metastasis carried the mutation $(\mathrm{p}=0.0067)$. Vascular invasion and ECE were not significantly correlated with the mutation. This is summarized in Graph 2.

To determine if the patient's age at diagnosis had an effect on BRAF mutation, the samples were also separated into groups based on being over or under 60 years of age (the median age of patients was $52.5 \pm 14$ ). There was no correlation between patient age and BRAF mutation status based on this age criterion.

Because, this study focused on fresh, frozen samples, there was little clinical follow-up data. In lieu of that data, 


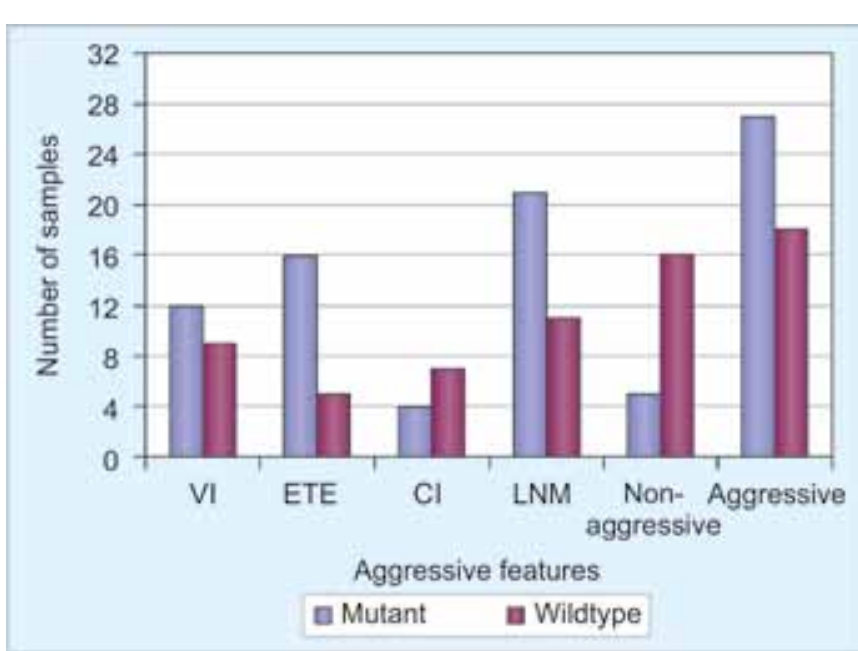

Graph 2: BRAF mutation testing results for pathological features of aggressiveness. Aggressive samples are those samples showing any of the four features. This category as well as ETE and LNM, were significantly correlated with the V600E BRAF mutation

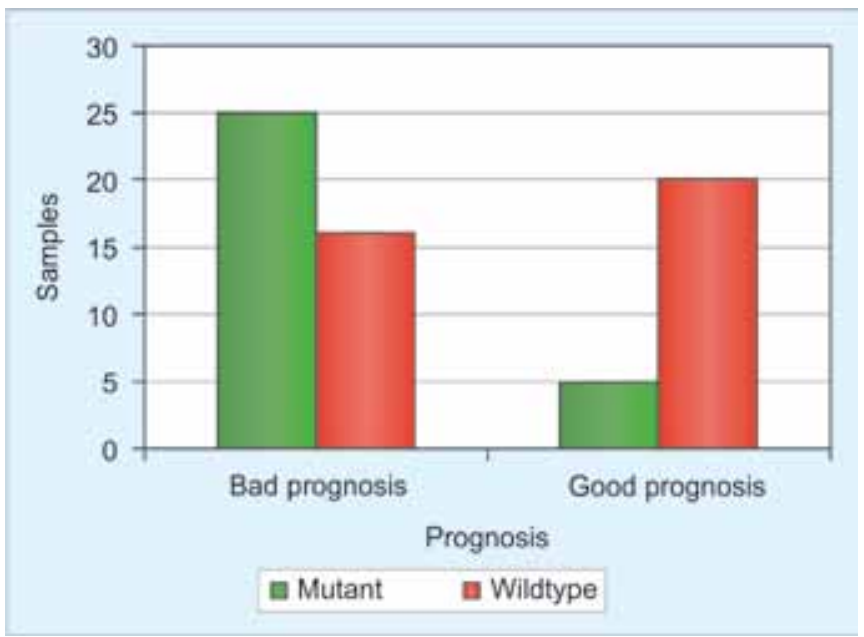

Graph 3: BRAF mutation testing as marker for prognosis. Poor prognosis was classified by having ETE, LNM, or VI. Good prognosis was classified by having $\mathrm{Cl}$ or none of the aggressive features. Poor prognosis was correlated to the BRAF mutation. V600E has $60 \%$ sensitivity and $80 \%$ specificity

samples that had any of the three pathological features (lymph node metastasis, extrathyroidal extension, vascular invasion) with relative survival rates less than $95 \%$ were categorized as having poor prognosis. Nonaggressive samples and those displaying only capsular invasion and nonaggressive samples were categorized as having a good prognosis. When categorized like this, the V600E BRAF mutation is significantly correlated with poor prognosis with a p-value less than 0.002 (Graph 3). However, there was only $60 \%$ sensitivity and $80 \%$ specificity of the assay to prognosis, making BRAF a poor singular marker for prognosis based on pathological aggressive features.

\section{DISCUSSION}

While the overriding feature of PTC is a benign prognosis, aggressive features, such as ETE, LNM, CI and vascular invasion may predict higher rates of recurrence or mortality. Testing for BRAF mutation may offer a molecular modality for the guidance of treatment in higher risk patients. The goal of this study was to determine if BRAF mutation testing can be used to predict any or all of the aggressive features for PTC. Detection of BRAF V600E mutation in all samples from this series was (48.5\%) agrees with majority of previous reports, falling in the 45 to $55 \%$ range.

Extrathyroidal extent is likely the most important of the aggressive features and the second most common (seer database). In the cohort for this study, 21 out of the 45 aggressive samples exhibited ETE. This result corroborates work done by Howell et al. ${ }^{19,20,22-24}$ The MAPK/ERK pathway regulates cell division. A mutation, like V600E, activating this pathway would cause unchecked growth, causing the cancer to extend to the surrounding tissue.

Lymph note metastasis is the most common of the aggressive features (SEER) and was also found to correlate with the V600E BRAF mutation in our study. This was the most common of the four pathological features, with 32 of the 45 aggressive patients containing this factor as well as the most common of the four pathological features according to the SEER statistics. Lymph note metastasis will prompt a regional neck dissection and may also require postoperative radioactive iodine ablation. Whether the BRAF mutation can predict which levels of the neck would be involved with metastases could be studied further.

With the two most common pathological aggressive features significantly correlated to the BRAF mutation, it follows that the samples displaying any of the four features were also found to be significantly correlated to the mutation. The correlation of LNM and ETE with the V600E BRAF mutation concurs with the meta-analysis aggregated by Kim. ${ }^{19}$ Vascular invasion and CI were not significantly correlated with BRAF in our study. While having the worst survival rate over 10 years, vascular invasion is the least common of the four pathological features for aggressiveness according to SEER. In the cohort for this study, however, 21 of the 45 samples exhibited vascular invasion. The mutation testing showed sensitivity to vascular invasion to be less than $60 \%$. Capsular invasion was the least common in this cohort of samples and had the highest survival rate over 10 years according to the SEER data. According to SEER data from 1998 to 2008, patients with capsular invasion in PTC had a relative survival rate of $98.5 \%$. Because of this survival rate, which is higher than the 10-year survival rate of all PTC patients in the SEER database between 1998 and 2008, this aggressive criterion was not used as a measure of poor prognosis. 
Advanced age at time of diagnosis also affects prognosis of the patient. Surveillance, epidemiology and end result data show that patients over 60 at time of diagnosis have a drop in survival after the 8 th year, and this drops the rate below the rate of overall PTC. Results show that BRAF mutation does not correlate with samples over 60 years of age at time of diagnosis. Therefore, the BRAF mutation testing is not hindered by age effects as an early marker for clinical aggressiveness.

This suggests that the MAPK signaling pathway is also being activated either upstream or downstream from BRAF. One cause of upstream activation may be the well-known ret proto oncogene/papillary thyroid cancer (RET/PTC) translocation, while activation of NFK maybe activated by other means downstream of BRAF. These other activating mechanisms of MAPK pathway are currently being studied and further examination is warranted.

\section{CONCLUSION}

The V600E BRAF mutation is correlated with samples having one or more of the four classic features for aggressiveness. This is primarily driven by the mutation correlating with the two most common of these features, extra-thyroidal extension and lymph node metastasis. When these features are used as a surrogate gauge for patient prognosis, the mutation test is shown to be unsuitable as a lone early marker for aggressiveness. With only $60 \%$ sensitivity and $80 \%$ specificity of the assay to poor prognosis based on three of the pathological features, the BRAF mutation test would overlook too many patients to be used as a lone marker for early aggressiveness.

\section{ACKNOWLEDGMENTS}

The authors would like to thank Sriharsha Gummadi for some of the sample preparation work as well as the staff of the Genomics Core at the Kimmel Cancer Center. This study was funded as part of the Kimmel Cancer Center at Thomas Jefferson University, Jefferson Medical College. The sponsors had no role in the study design, data collection and analysis, decision to publish or preparation of the manuscript. This paper was presented at the 8th International Head and Neck Society Meeting, July 25th 2012, Toronto, Ontario, Canada.

\section{AUTHOR CONTRIBUTIONS}

Drs Wynne and Quong had full access to all data in this study and take responsibility for integrity of data and accuracy of analysis. Study concept and design: Wynne, Quong, Pribitkin, Duddy, Scott, Curry. Data acquisition: Wynne, Quong, Scott. Analysis and interpretation of data: Wynne, Quong, Scott. Drafting of Manuscript: Wynne, Quong, Pribitkin, Curry, Cognetti. Critical revision: Wynne, Quong, Pritbitkin, Curry, Cognetti, Rosen. Obtained funding: Quong and Pribitkin. Administrative, technical and material support: Duddy and Scott. Study supervision: Quong and Pribitkin.

\section{REFERENCES}

1. Mathur A, Moses W, Rahbari R, Khanafshar E, Duh QY, Clark $\mathrm{O}$, Kebebew E. Higher rate of BRAF mutation in papillary thyroid cancer over time: a single-institution study. Cancer 2011;117:4390-4395.

2. Lin KL, Wang OC, Zhang XH, Dai XX, Hu XQ, Qu JM. The BRAF mutation is predictive of aggressive clinicopathological characteristics in papillary thyroid microcarcinoma. Ann Surg Oncol 2010;17:3294-3300.

3. Yu XM, Wan Y, Sippel RS, Chen H. Should all papillary thyroid microcarcinomas be aggressively treated? An analysis of 18,445 cases. Ann Surg 2011;254:653-660.

4. Faquin WC: The thyroid gland: recurring problems in histologic and cytologic evaluation. Arch Pathol Lab Med 2008;132:622-632.

5. Lin HY, Davis FB, Gordinier JK, Martino LJ, Davis PJ. Thyroid hormone induces activation of mitogen-activated protein kinase in cultured cells. Am J Physiol 1999;276:C1014-1024.

6. Caronia LM, Phay JE, Shah MH. Role of BRAF in thyroid oncogenesis. Clin Cancer Res 2011;17:7511-7517.

7. Sullivan RJ, Flaherty KT. BRAF in Melanoma: pathogenesis, diagnosis, inhibition and resistance. J Skin Cancer 2011;2011:423239.

8. Kalady MF, Dejulius KL, Sanchez JA, Jarrar A, Liu X, Manilich E, Skacel M, Church JM. BRAF mutations in colorectal cancer are associated with distinct clinical characteristics and worse prognosis. Dis Colon Rectum 2012;55:128-133.

9. Knauf JA, Sartor MA, Medvedovic M, Lundsmith E, Ryder M, Salzano M, Nikiforov YE, Giordano TJ, Ghossein RA, Fagin JA. Progression of BRAF-induced thyroid cancer is associated with epithelial-mesenchymal transition requiring concomitant MAP kinase and TGFbeta signaling. Oncogene 2011;30:3153-3162.

10. Patel A, Klubo-Gwiezdzinska J, Hoperia V, Larin A, Jensen K, Bauer A, Vasko V. BRAF (V600E) mutation analysis from May-Grunwald Giemsa-stained cytological samples as an adjunct in identification of high-risk papillary thyroid carcinoma. Endocr Pathol 2011;22:195-199.

11. Nam SY, Han BK, Ko EY, Kang SS, Hahn SY, Hwang JY, Nam MY, Kim JW, Chung JH, Oh YL, et al. BRAF V600E mutation analysis of thyroid nodules needle aspirates in relation to their ultrasonographic classification: a potential guide for selection of samples for molecular analysis. Thyroid 2010;20:273-279.

12. Lee EJ, Song KH, Kim DL, Jang YM, Hwang TS, Kim SK. The BRAF (V600E) mutation is associated with malignant ultrasonographic features in thyroid nodules. Clin Endocrinol (Oxf) 2011;75:844-850.

13. Xing M. BRAF mutation in papillary thyroid cancer: pathogenic role, molecular bases and clinical implications. Endocr Rev 2007;28:742-762.

14. Ahn D, Park JS, Sohn JH, Kim JH, Park SK, Seo AN, Park JY. BRAF (V600E) mutation does not serve as a prognostic factor in Korean patients with papillary thyroid carcinoma. Auris Nasus Larynx 2012;39:198-203. 
15. Sassolas G, Hafdi-Nejjari Z, Ferraro A, Decaussin-Petrucci M, Rousset B, Borson-Chazot F, Borbone E, Berger N, Fusco A. Oncogenic alterations in papillary thyroid cancers of young patients. Thyroid 2012;22:17-26.

16. Wang W, Zhao W, Wang H, Teng X, Chen X, Li Z, Yu X, Fahey TJ 3rd, Teng L. Poorer prognosis and higher prevalence of BRAF (V600E) mutation in synchronous bilateral papillary thyroid carcinoma. Ann Surg Oncol 2011;27:27.

17. Paulson L, Shindo M, Schuff K, Corless C: The role of molecular markers and tumor histological type in central lymph node metastasis of papillary thyroid carcinoma. Arch Otolaryngol Head Neck Surg 2012;138:44-49.

18. Basolo F, Torregrossa L, Giannini R, Miccoli M, Lupi C, Sensi E, Berti P, Elisei R, Vitti P, Baggiani A, et al. Correlation between the BRAF V600E mutation and tumor invasiveness in papillary thyroid carcinomas smaller than 20 millimeters: analysis of 1060 cases. J Clin Endocrinol Metab 2010;95:41974205.

19. Kim SJ, Lee KE, Myong JP, Park JH, Jeon YK, Min HS, Park SY, Jung KC, Koo do H, Youn YK. BRAF V600E mutation is associated with tumor aggressiveness in papillary thyroid cancer. World J Surg 2012;36:310-317.
20. Howell GM, Carty SE, Armstrong MJ, Lebeau SO, Hodak SP, Coyne C, Stang MT, McCoy KL, Nikiforova MN, Nikiforov YE, et al. Both BRAF V600E mutation and older age ( $\geq 65$ years) are associated with recurrent papillary thyroid cancer. Ann Surg Oncol 2011;18:3566-3571.

21. Kim TH, Park YJ, Lim JA, Ahn HY, Lee EK, Lee YJ, Kim KW, Hahn SK, Youn YK, Kim KH, et al. The association of the BRAF (V600E) mutation with prognostic factors and poor clinical outcome in papillary thyroid cancer: a meta-analysis. Cancer 2012;118:1764-1773.

22. Colanta A, Lin O, Tafe L, Ghossein R, Nafa K, Mitchell T, Ladanyi M, Arcila M. BRAF mutation analysis of fine-needle aspiration biopsies of papillary thyroid carcinoma: impact on diagnosis and prognosis. Acta Cytol 2011;55:563-569.

23. Filicori F, Keutgen XM, Buitrago D, AlDailami H, Crowley M, Fahey TJ 3rd, Zarnegar R. Risk stratification of indeterminate thyroid fine-needle aspiration biopsy specimens based on mutation analysis. Surgery 2011;150:1085-1091.

24. Yip L, Nikiforova MN, Carty SE, Yim JH, Stang MT, Tublin MJ, Lebeau SO, Hodak SP, Ogilvie JB, Nikiforov YE. Optimizing surgical treatment of papillary thyroid carcinoma associated with BRAF mutation. Surgery 2009;146:1215-1223. 